\title{
Test-space characterizations of some classes of Banach spaces
}

\author{
M. I. Ostrovskii
}

November 5, 2018

\begin{abstract}
Let $\mathcal{P}$ be a class of Banach spaces and let $T=\left\{T_{\alpha}\right\}_{\alpha \in A}$ be a set of metric spaces. We say that $T$ is a set of test-spaces for $\mathcal{P}$ if the following two conditions are equivalent: (1) $X \notin \mathcal{P}$; (2) The spaces $\left\{T_{\alpha}\right\}_{\alpha \in A}$ admit uniformly bilipschitz embeddings into $X$.

The first part of the paper is devoted to a simplification of the proof of the following test-space characterization obtained in M. I. Ostrovskii [Different forms of metric characterizations of classes of Banach spaces, Houston J. Math., to appear]:

For each sequence $\left\{X_{m}\right\}_{m=1}^{\infty}$ of finite-dimensional Banach spaces there is a sequence $\left\{H_{n}\right\}_{n=1}^{\infty}$ of finite connected unweighted graphs with maximum degree 3 such that the following conditions on a Banach space $Y$ are equivalent: (A) $Y$ admits uniformly isomorphic embeddings of $\left\{X_{m}\right\}_{m=1}^{\infty}$; (B) $Y$ admits uniformly bilipschitz embeddings of $\left\{H_{n}\right\}_{n=1}^{\infty}$.

The second part of the paper is devoted to the case when $\left\{X_{m}\right\}_{m=1}^{\infty}$ is an increasing sequence of spaces. It is shown that in this case the class of spaces given by $(\mathbf{A})$ can be characterized using one test-space, which can be chosen to be an infinite graph with maximum degree 3 .
\end{abstract}

2010 Mathematics Subject Classification: Primary: 46B07; Secondary: 05C12, 46B85, 54E35

\section{Contents}

1 Introduction 1

2 Simplification of the proof on metric characterizations of classes with excluded subspaces

3 Metric characterization with one test-space

4 Characterization in terms of an infinite graph with maximum degree 3

\section{Introduction}

Embeddings of metric spaces into Banach spaces play an important role in Computer Science (see, for example, WS11, Chapter 15]) and in Topology (see [Yu06]). In connection with problems of embeddability of metric spaces into Banach spaces it 
would be interesting to find metric characterizations of well-known classes of Banach spaces. By metric characterizations we mean characterizations which can be tested on an arbitrary metric space. So, in a metric characterization only distances between elements of the space are involved, and no linear combinations of any kind can be used. At this point it seems to be unclear: how to define the notion of a metric characterization which would be the most useful for applications in the theory of metric embeddings? One can try to define a metric characterization in the following way: a metric characterization is a set of formulas understood as in logic (see [Han77, p. 19] for a definition of a first-order formula). Some of the variables in the formulas are elements in an unknown metric space $X$ (so the formulas make sense for an arbitrary metric space $X$ ). We say that such set of formulas characterizes a class $\mathcal{P}$ of Banach spaces if the following conditions are equivalent for a Banach space $X$ :

- $X \in \mathcal{P}$

- All of the formulas of the set hold for $X$.

Metric characterizations which we are going to study in this paper use the following definition.

Definition 1.1. Let $0 \leq C<\infty$. A map $f:\left(A, d_{A}\right) \rightarrow\left(Y, d_{Y}\right)$ between two metric spaces is called $C$-Lipschitz if

$$
\forall u, v \in A \quad d_{Y}(f(u), f(v)) \leq C d_{A}(u, v) .
$$

A map $f$ is called Lipschitz if it is $C$-Lipschitz for some $0 \leq C<\infty$. For a Lipschitz map $f$ we define its Lipschitz constant by

$$
\operatorname{Lip} f:=\sup _{d_{A}(u, v) \neq 0} \frac{d_{Y}(f(u), f(v))}{d_{A}(u, v)} .
$$

Let $1 \leq C<\infty$. A map $f: A \rightarrow Y$ is called a $C$-bilipschitz embedding if there exists $r>0$ such that

$$
\forall u, v \in A \quad r d_{A}(u, v) \leq d_{Y}(f(u), f(v)) \leq r C d_{A}(u, v) .
$$

A bilipschitz embedding is an embedding which is $C$-bilipschitz for some $1 \leq C<\infty$. The smallest constant $C$ for which there exist $r>0$ such that (11) is satisfied is called the distortion of $f$. A set of embeddings is called uniformly bilipschitz if they have uniformly bounded distortions.

Remark 1.2. Linear embeddings $T_{i}: A_{i} \rightarrow Y$ of Banach (or normed) spaces into a Banach (normed) space are uniformly bilipschitz if and only if

$$
\sup _{i}\left(\left\|T_{i}\right\| \cdot \|\left. T_{i}^{-1}\right|_{T_{i}\left(A_{i}\right)}||\right)<\infty .
$$

Such embeddings $T_{i}$ are called uniformly isomorphic. 
Remark 1.3. The definition of a metric characterization suggested above does not seem to be completely satisfactory. It includes trivial (in a certain sense) characterizations of the type: A Banach space is nonreflexive if and only if it contains a (separable) subset which is bilipschitz equivalent to a nonreflexive separable Banach space. (The validity of this characterization is a consequence of the following well-known facts: (1) Each nonreflexive Banach space contains a separable nonreflexive subspace. This fact follows, for example, from the Eberlein-Shmulian theorem [DS58, Theorem V.6.1]; (2) If a Banach space $Y$ admits a bilipschitz embedding of a (separable) nonreflexive Banach space $X$, then $Y$ is nonreflexive, see HM82, Lemma 3.1] or [BL00, Theorem 7.9].)

At this point it is not clear how to define a metric characterization in such a way that, on one hand, all interesting examples are included, but, on the other hand, characterizations like the trivial characterization of nonreflexivity mentioned in Remark 1.3 are excluded. We shall focus on one of the classes of metric characterizations which is known to be interesting (see Bau07, Bau09+, Bou86, BMW86, JS09, MN08, Ost11a, Ost11d, Pis86]). We mean metric characterizations of the following type.

Definition 1.4. Let $\mathcal{P}$ be a class of Banach spaces and let $T=\left\{T_{\alpha}\right\}_{\alpha \in A}$ be a set of metric spaces. We say that $T$ is a set of test-spaces for $\mathcal{P}$ if the following two conditions are equivalent

1. $X \notin \mathcal{P}$.

2. The spaces $\left\{T_{\alpha}\right\}_{\alpha \in A}$ admit uniformly bilipschitz embeddings into $X$.

Remark 1.5. We use $X \notin \mathcal{P}$ in condition 1 of Definition 1.4 rather than $X \in \mathcal{P}$ for terminological reasons: we would like to use terms "test-spaces for reflexivity, superreflexivity, etc." rather than "test-spaces for nonreflexivity, nonsuperreflexivity, etc."

Remark 1.6. One can introduce the notion of test-spaces differently, requiring, for example: "at least one of the spaces $\left\{T_{\alpha}\right\}_{\alpha \in A}$ admits a bilipschitz embedding into $X$ ". However, this version of test-space characterizations includes the trivial characterization of reflexivity mentioned in Remark 1.3. Another reason why we have chosen the introduced in Definition 1.4 notion of test-spaces is: many important known characterizations are of this form (see Bau07, Bau09+, Bou86, BMW86, JS09, MN08, Ost11a, Ost11d, Pis86]).

\section{Simplification of the proof on metric characterizations of classes with excluded subspaces}

The main purpose of this section is to give a simpler proof of the following result of Ost11d: 
Theorem 2.1 ([st11d]). For each sequence $\left\{X_{m}\right\}_{m=1}^{\infty}$ of finite-dimensional Banach spaces there exists a sequence $\left\{H_{n}\right\}_{n=1}^{\infty}$ of finite connected unweighted graphs with maximum degree 3 such that the following conditions on a Banach space $Y$ are equivalent:

- $Y$ admits uniformly isomorphic embeddings of $\left\{X_{m}\right\}_{m=1}^{\infty}$.

- $Y$ admits uniformly bilipschitz embeddings of $\left\{H_{n}\right\}_{n=1}^{\infty}$.

Everywhere in this paper we consider graphs as metric spaces with their shortest path metric: the distance between two vertices is the length of the shortest path between them. In some cases we consider weighted graphs with some positive weights assigned to their edges. In such a case the length of the path is the sum of weights of edges included in it. For graphs with no weights (sometimes we emphasize this by calling them unweighted graphs) the length of a path is the number of edges in it (this corresponds to the case when all weights are equal to 1).

Remark 2.2. For the reasons explained in Ost11d we restrict our attention to the case $\sup _{m} \operatorname{dim} X_{m}=\infty$.

Our purpose is to simplify the proof of the step which has the longest proof in Ost11d]. We shall also present other steps of the proof, in a more general form than in Ost11d]. The reason for doing so is that we need these steps in the more general form later in the paper. Recall some standard definitions.

Definition 2.3. Let $\alpha>0$. We say that a subset $A$ in a metric space $(X, d)$ is $\alpha$-dense in a subset $B \subset X$ if $A \subset B$ and

$$
\forall x \in B \exists y \in A d(x, y) \leq \alpha .
$$

A subset $D$ in a metric space $(X, d)$ is called $\alpha$-separated if $d(x, y) \geq \alpha$ for each $x, y \in D, x \neq y$.

If $A$ and $B$ are subsets in a metric space $(X, d)$, we let

$$
d(A, B)=\inf \{d(x, y): x \in A, y \in B\} .
$$

First we introduce an approximate description of convex sets in Banach spaces using unweighted graphs. Let $C$ be a convex set in a Banach space $X, \alpha, \beta>0$, and let $V$ be an $\alpha$-separated $\beta$-dense subset of $C$.

Remark 2.4. It is easy to see that such subset $V$ does not exist if $\beta<\frac{\alpha}{2}$ and $\operatorname{diam} C>\beta$. In this paper only the case where $\beta \geq \alpha$ is considered. In this case the existence is immediate from Zorn's lemma.

Definition 2.5. Let $G$ be the graph whose vertex set is $V$ and whose edge set is defined in the following way: vertices $u, v \in V$ are joined by an edge if and only if $\|u-v\| \leq 3 \beta$. The graph $G$ is called a $(C, \alpha, \beta)$-graph. If $\alpha=\beta, G$ is called a $(C, \alpha)$-graph. 
It is easy to check that $G$ is not uniquely determined by $C, \alpha$, and $\beta$, but for our purposes it does not matter which of $(C, \alpha, \beta)$-graphs we pick. We endow the vertex set $V$ of $G$ with its shortest path metric $d_{G}$.

Lemma 2.6. The natural embedding $f:\left(V, d_{G}\right) \rightarrow(X,\|\cdot\|)$ is bilipschitz with distortion $\leq \max \left\{3, \frac{3 \beta}{\alpha}\right\}$. More precisely,

$$
\operatorname{Lip}(f) \leq 3 \beta \text { and } \operatorname{Lip}\left(\left.f^{-1}\right|_{f(V)}\right) \leq \max \left\{\frac{1}{\beta}, \frac{1}{\alpha}\right\} .
$$

Proof. The inequality $\operatorname{Lip}(f) \leq 3 \beta$ follows immediately from the fact that adjacent vertices in $G$ are at distance $\leq 3 \beta$ in $X$, and the definition of the shortest path metric.

To prove the inequality for $\operatorname{Lip}\left(f^{-1}\right)$ we consider two distinct vertices $u, v \in V$, write $\|u-v\|=d \beta$ for some $d>0$, and consider two cases:

Case A. $d \leq 3$. In such a case $d_{G}(u, v)=1$. Since $\|u-v\| \geq \alpha$, we have

$$
\frac{d_{G}(u, v)}{\|u-v\|} \leq \frac{1}{\alpha} .
$$

Case B. $d>3$. In this case, and even in a wider case $d>2$, we show that

$$
d_{G}(u, v) \leq\lfloor d\rfloor-1
$$

and therefore

$$
\frac{d_{G}(u, v)}{\|u-v\|} \leq \frac{\lfloor d\rfloor-1}{d \beta} \leq \frac{1}{\beta} .
$$

We prove the inequality (2) by induction starting with $2<d \leq 3$. In this case $d_{G}(u, v)=1$ and so it is clear that (2) is satisfied.

Suppose that we have proved the inequality (2) for $2<d \leq n$. Let us show that this implies the inequality for $n<d \leq n+1$. We do this as follows:

Consider the vertex $\widetilde{u}$ lying on the line segment joining $u$ and $v$ at distance $2 \beta$ from $u$. Since $\widetilde{u} \in C$ (this is the point where we use the convexity of $C$ ), there is $w \in V$ satisfying $\|w-\widetilde{u}\| \leq \beta$.

By the triangle inequality, we have $\|w-u\| \leq 3 \beta$ and $\|w-v\| \leq(d-1) \beta$. The first inequality implies $d_{G}(w, u)=1$. Applying the triangle inequality and the Induction Hypothesis, we get $d_{G}(u, v) \leq d_{G}(w, v)+1 \leq(\lfloor d-1\rfloor-1)+1=\lfloor d\rfloor-1$. (We cannot apply the Induction Hypothesis if $\|w-v\| \leq 2 \beta$, but in this case the result is also easy to verify.)

If $X$ is a Banach space, we use the notation $B_{X}(r), r>0$, for $\{x \in X:\|x\| \leq r\}$. The unit ball $B_{X}(1)$ is also denoted by $B_{X}$. Observe that if $X$ is finite-dimensional (and $\beta \geq \alpha),\left(B_{X}(r), \alpha, \beta\right)$-graphs are finite.

The next step in the proof of Theorem 2.1] in Ost11d] is the following lemma (in Ost11d the lemma is stated in slightly less general form). 
Lemma 2.7. If $\left\{X_{m}\right\}_{m=1}^{\infty}$ are finite-dimensional Banach spaces, and a Banach space $Y$ admits uniformly bilipschitz embeddings of a collection of $\left(B_{X_{m}}(n), \alpha(n), \beta(n)\right)$ graphs $m, n \in \mathbb{N}$, where $\alpha(n) \leq \beta(n), \lim _{n \rightarrow \infty} \alpha(n)=0, \lim _{n \rightarrow \infty} \beta(n)=0$, and $\sup _{n} \frac{\beta(n)}{\alpha(n)}<\infty$, then $\left\{X_{m}\right\}_{m=1}^{\infty}$ are uniformly isomorphic to subspaces of $Y$.

Lemma 2.7 can be derived from the following discretization result.

Theorem 2.8. For each finite-dimensional Banach space $X$ and each $\gamma>0$ there exists $\varepsilon>0$ such that for each bilipschitz embedding $L$ of an $\varepsilon$-dense subset of $B_{X}$, with the metric inherited from $X$, into a Banach space $Y$ there is a linear embedding $T: X \rightarrow Y$ such that $(1-\gamma)\|T\| \cdot\left\|T^{-1}\right\| \leq$ (the distortion of $\left.L\right)$.

This theorem goes back to Ribe [Rib76], a new proof of the essential ingredients was found by Heinrich-Mankiewicz HM82, versions of these proofs are presented in [BL00]. The first explicit bound on $\varepsilon$ was found by Bourgain Bou87. Bourgain's proof was simplified and explained by Begun Beg99 and Giladi-Naor-Schechtman GNS11. We recommend everyone who would like to study this result to start by reading GNS11.

It is clear that the fact that the ball in Theorem 2.8 has radius 1 plays no role. To derive Lemma 2.7 from Theorem 2.8 we observe that the vertex set of a $\left(B_{X_{m}}(n), \alpha(n), \beta(n)\right)$-graph is a $\beta(n)$-dense subset of $B_{X_{m}}(n)$. Furthermore, by Lemma 2.6, the graph distance on this set is $\max \left\{3, \frac{3 \beta(n)}{\alpha(n)}\right\}$-equivalent to the metric inherited from $X$. This proves Lemma 2.7.

Lemmas 2.6 and 2.7 show that a Banach space $Y$ admits uniformly isomorphic embeddings of $X_{m}$ if and only if $Y$ admits uniformly bilipschitz embeddings of some (or any) collection of $\left(B_{X_{m}}(n), \alpha(n), \beta(n)\right)$-graphs $m, n \in \mathbb{N}$, where $\alpha(n) \leq \beta(n)$, $\lim _{n \rightarrow \infty} \alpha(n)=0, \lim _{n \rightarrow \infty} \beta(n)=0$, and $\sup _{n} \frac{\beta(n)}{\alpha(n)}<\infty$. This statement does not complete the proof of Theorem 2.1 (even of a weaker version of it, with 3 replaced by any other uniform bound on degrees of $\left.\left\{H_{n}\right\}_{n=1}^{\infty}\right)$. In fact, it is easy to see that if $\sup _{m} \operatorname{dim} X_{m}=\infty$, the degrees of any collection of $\left(B_{X_{m}}(n), \alpha(n), \beta(n)\right)$ graphs, $m, n \in \mathbb{N}$, where $\alpha(n) \leq \beta(n), \lim _{n \rightarrow \infty} \alpha(n)=0, \lim _{n \rightarrow \infty} \beta(n)=0$, and $\sup _{n} \frac{\beta(n)}{\alpha(n)}<\infty$, are unbounded.

Observation 2.9. Since we assumed $\sup _{m} \operatorname{dim} X_{m}=\infty$, each Banach space admitting uniformly isomorphic embeddings of $X_{m}$ has to be infinite-dimensional. For this reason, in order to prove Theorem 2.1 it suffices to show that for each finitedimensional Banach space $X$, each $\left(B_{X}(r), \delta\right)$-graph $G(0<\delta<r<\infty)$, and each infinite-dimensional Banach space $Z$ containing $X$ as a subspace, there exist a graph $H$ and bilipschitz embeddings $\psi: G \rightarrow H$ and $\varphi: H \rightarrow Z$, such that distortions of $\psi$ and $\varphi$ are bounded from above by absolute constants and the maximum degree of $H$ is 3. It is clear that it is enough to consider the case $\delta=1$.

Remark 2.10. Observation 2.9 is the main step towards simplification of the proof of Theorem 2.1 given in Ost11d: in Ost11d the graph $H$ was embedded into $X$ 
(if $\operatorname{dim} X \geq 3$ ). This is substantially more difficult and, as we see from Observation 2.9, is not needed to prove Theorem 2.1. However, the proof of [Ost11d, Section 4] could be of independent interest, see Remark 2.12 below.

The construction of $H$ which we use is the same as in Ost11d: We introduce the graph $M G$ as the following "expansion" of $G$ : we replace each edge in $G$ by a path of length $M$. It is clear that the graph $M G$ is well-defined for each $M \in \mathbb{N}$. In our construction of $H$ the number $M$ will be chosen to be much larger than the number of edges of $G$. We use the term long paths for the paths of length $M$ which replace edges of $G$. Next step in the construction of $H$ : For each vertex $v$ of $G$, we introduce a path $p_{v}$ in the graph $H$ whose length is equal to the number of edges of $G$, we call each such path a short path. At the moment these paths do not interact. We continue our construction of $H$ in the following way. We label vertices of short paths in a monotone way by long paths. "In a monotone way" means that the first vertex of each short path corresponds to the long path $p_{1}$, the second vertex of each short path corresponds to the long path $p_{2}$ etc. We complete our construction of $H$ introducing, for a long path $p$ in $M G$ corresponding to an edge $u v$ in $G$, a path of the same length in $H$ (we also call it long) which joins those vertices of the short paths $p_{u}$ and $p_{v}$ which have label $p$. There is no further interaction between short and long paths in $H$. It is obvious that the maximum degree of $H$ is 3 .

It remains to define embeddings $\psi$ and $\varphi$ and to estimate their distortions.

To define $\psi$ we pick a long path $p$ in $M G$ (in an arbitrary way) and map each vertex $u$ of $G$ onto the vertex in $H$ having label $p$ in the short path $p_{u}$ corresponding to $u$. The estimates for $\operatorname{Lip}(\psi)$ and $\operatorname{Lip}\left(\psi^{-1}\right)$ given below are taken from Ost11d. We reproduce them because they do not take much space.

We have $\operatorname{Lip}(\psi) \leq 2 e(G)+M$, where $e(G)$ is the number of edges of $G$. In fact, to estimate the Lipschitz constant it suffices to find an estimate from above for the distances in $H$ between $\psi(u)$ and $\psi(v)$ where $u$ and $v$ are adjacent vertices of $G$. To see that $2 e(G)+M$ provides the desired estimate we consider the following three-stage walk from $\psi(u)$ from $\psi(v)$ :

- We walk from $\psi(u)$ along the short path $p_{u}$ to the vertex labelled by the long path corresponding to the edge $u v$ in $G$.

- Then we walk along the corresponding long path to its end in $p_{v}$.

- We conclude the walk with the piece of the short path $p_{v}$ which we need to traverse in order to reach $\psi(v)$.

We claim that $\operatorname{Lip}\left(\psi^{-1}\right) \leq M^{-1}$. This gives an absolute upper bound for the distortion of $\psi$ provided the quantity $e(G)$ is controlled by $M$, we need $M$ to be much larger than $e(G)$ only if we would like to make the distortion close to 1 . To prove $\operatorname{Lip}\left(\psi^{-1}\right) \leq M^{-1}$ we let $\psi(u)$ and $\psi(v)$ be two vertices of $\psi(V(G))$. We need to estimate $d_{G}(u, v)$ from below in terms of $d_{H}(\psi(u), \psi(v))$. Let

$$
P=\psi(u), w_{1}, \ldots, w_{n-1}, \psi(v)
$$


be one of the shortest $\psi(u) \psi(v)$-paths in $H$. Let $u, u_{1}, \ldots, u_{k-1}, v$ be those vertices of $G$ for which the path $P$ visits the corresponding short paths $p_{u}, p_{u_{1}}, \ldots, p_{u_{k-1}}, p_{v}$. We list $u_{1}, \ldots, u_{k-1}$ in the order of visits. It is clear that in such a case the sequence $u, u_{1}, \ldots, u_{k-1}, v$ is a $u v$-walk in $G$. Therefore $d_{G}(u, v) \leq k$. On the other hand, in $H$, to move from one short path to another, one has to traverse at least $M$ edges, therefore $d_{H}(\psi(u), \psi(v)) \geq k M$. This implies $\operatorname{Lip}\left(\psi^{-1}\right) \leq M^{-1}$.

Our next purpose is to introduce $\varphi: H \rightarrow Z$. First we prove (Lemma 2.11 below) that there is a bilipschitz embedding of $M G$ into some finite-dimensional subspace $W$ of $Z$ with distortion bounded by an absolute constant.

It is convenient to handle all $M \in \mathbb{N}$ simultaneously by considering the following thickening of the graph $G$ (see [Gro93, Section 1.B] for the general notion of thickening). For each edge $u v$ in $G$ we join $u$ and $v$ with a set isometric to [0,1], we denote this set $t(u v)$. The thickening $T G$ of $G$ is the union of all sets $t(u v)$ (such sets can intersect at their ends only) with the distance between two points defined as the length of the shortest curve joining the points.

Lemma 2.11. If a finite unweighted graph $G$ endowed with its graph distance admits a bilipschitz embedding $\tau$ into a finite-dimensional Banach space $X$, then the graph $T G$ admits a bilipschitz embedding $f$ into any infinite-dimensional Banach space $Z$ containing $X$ as a subspace, and the distortion of $f$ is bounded in terms of the distortion of $\tau$ and some absolute constants.

Remark 2.12. In Ost11d, Section 4] a stronger result was proved, namely, it was proved that in the case where $\operatorname{dim} X \geq 3$, the bilipschitz embedding $f$ whose existence is claimed in Lemma 2.11 can be required to map $T G$ into $X$. As we shall see, this result is not needed for Theorem 2.1. However, it could be of independent interest.

Proof of Lemma 2.11. We may assume without loss of generality that $\operatorname{Lip}\left(\tau^{-1}\right)=1$, that is, $\|\tau(u)-\tau(v)\| \geq d_{G}(u, v)$. We construct a bilipschitz embedding of $T G$ into an (arbitrary) Banach space $W$ containing $X$ as a subspace and satisfying $\operatorname{dim}(W / X)=e(G)$, where $e(G)$ is the number of edges in $G$ and $W / X$ is the quotient space.

We find an Auerbach basis in $W / X$. Recall the definition. Let $\left\{x_{i}\right\}_{i=1}^{n}$ be a basis in an $n$-dimensional Banach space $Y$, its biorthogonal functionals are defined by $x_{i}^{*}\left(x_{j}\right)=\delta_{i j}$ (Kronecker delta). The basis $\left\{x_{i}\right\}_{i=1}^{n}$ is called an Auerbach basis if $\left\|x_{i}\right\|=\left\|x_{i}^{*}\right\|=1$ for all $i \in\{1, \ldots, n\}$. This notion goes back to [Aue30]. See Ost11b, Section 2] and [Pli95] for historical comments and proofs.

Since the cardinality of the Auerbach basis is equal to the number of edges in $G$, we label its elements by edges. Also we lift the elements of this Auerbach basis into $W$. Since $W$ is finite-dimensional, we may assume that the norms of the lifted elements are also equal to 1 . We use the notation $\left\{e_{u v}\right\}_{u v \in E(G)}$ for the lifted elements 
of the Auerbach basis and the notation $\left\{e_{u v}^{*}\right\}_{u v \in E(G)}$ for its biorthogonal system. It is clear that $\left\{e_{u v}^{*}\right\}_{u v \in E(G)}$ may be regarded as elements of $W^{*}$.

If $u v$ is an edge in $G$, we map $t(u v)$ onto the concatenation of two line segments in $W \subset Z$, namely, onto the concatenation of $\left[\tau(u), \frac{\tau(u)+\tau(v)}{2}+e_{u v}\right]$ and $\left[\frac{\tau(u)+\tau(v)}{2}+e_{u v}, \tau(v)\right]$. More precisely, we map the point in $t(u v)$ which is at distance $\alpha \in[0,1]$ from $u$ onto the point

$$
\alpha \tau(v)+(1-\alpha) \tau(u)+\min \{2 \alpha, 2(1-\alpha)\} e_{u v} .
$$

It is easy to check that such points cover the concatenation of the line segments $\left[\tau(u), \frac{\tau(u)+\tau(v)}{2}+e_{u v}\right]$ and $\left[\frac{\tau(u)+\tau(v)}{2}+e_{u v}, \tau(v)\right]$. We denote this map by $f: T G \rightarrow$ $W$.

We claim that $f$ is a bilipschitz embedding and its distortion is bounded in terms of distortion of $\tau$ and some absolute constant. To estimate $\operatorname{Lip}(f)$ observe that the derivative of the function in (3) with respect to $\alpha$ is $\tau(v)-\tau(u) \pm 2 e_{u v}$ (at points where the derivative is defined). Hence $\operatorname{Lip}(f) \leq \operatorname{Lip}(\tau)+2$.

To estimate the Lipschitz constant of $f^{-1}$, we need to estimate from above the quotient

$$
\frac{d_{T G}(x, y)}{\|f(x)-f(y)\|}
$$

where $x, y \in T G$. Let $\alpha$ be the distance in $T G$ from $x \in t(u v)$ to $u$ and let $\beta$ be the distance from $y \in t(w z)$ to $w$. We may choose our notation in such a way that $\alpha, \beta \leq \frac{1}{2}$. Let $D=d_{T G}(x, y)$.

First we consider the case when the edges $u v$ and $w z$ are different. We have $d_{T G}(u, w) \geq D-\alpha-\beta$. Thus $\|f(u)-f(w)\|=\|\tau(u)-\tau(w)\| \geq D-\alpha-\beta$ and $\|f(x)-f(y)\| \geq D-(\operatorname{Lip}(\tau)+3)(\alpha+\beta)$. Here we use the fact that, since $\operatorname{Lip}(f) \leq \operatorname{Lip}(\tau)+2$, we have $\|f(x)-f(u)\| \leq(\operatorname{Lip}(\tau)+2) \alpha$ and $\|f(y)-f(w)\| \leq$ $(\operatorname{Lip}(\tau)+2) \beta$. On the other hand $\|f(x)-f(y)\| \geq e_{u v}^{*}(f(x)-f(y))=e_{u v}^{*}(f(x)-$ $f(u))+e_{u v}^{*}(f(u)-f(y))=e_{u v}^{*}(f(x)-f(u))=2 \alpha$ (we use $u v \neq w z$, the definition of $\alpha$ and the fact that $\left\{e_{u v}\right\}$ is a lifted Auerbach basis in $\left.W / X\right)$. Similarly we get $\|f(x)-f(y)\| \geq 2 \beta$. Therefore

$$
\frac{d_{T G}(x, y)}{\|f(x)-f(y)\|} \leq \min \left\{\frac{D}{\max \{0, D-(\operatorname{Lip}(\tau)+3)(\alpha+\beta)\}}, \frac{D}{2 \alpha}, \frac{D}{2 \beta}\right\}
$$

It is easy to see that the minimum in this inequality is bounded from above in terms of $\operatorname{Lip}(\tau)$ and an absolute constant. If $\alpha=0$, or $\beta=0$, or both, we modify this argument in a straightforward way.

It remains to consider the case where $x, y \in t(u v)$. Let $d_{T G}(x, u)=\alpha$ and 
$d_{T G}(y, u)=\beta$, so $d_{T G}(x, y)=|\alpha-\beta|$. It is easy to see that

$$
\left|e_{u v}^{*}(f(x)-f(y))\right|= \begin{cases}2|\alpha-\beta| & \text { if } \alpha \text { and } \beta \text { are on the same side of } \frac{1}{2} \\ 2|1-\alpha-\beta| & \text { otherwise. }\end{cases}
$$

In the former case we get

$$
\frac{d_{T G}(x, y)}{\|f(x)-f(y)\|} \leq \frac{1}{2}
$$

If the latter case we use

$$
f(x)-f(y)=(\alpha-\beta)(\tau(v)-\tau(u)) \pm 2(1-\alpha-\beta) e_{u v} .
$$

We get from here that

$$
\|f(x)-f(y)\| \geq \max \{2|1-\alpha-\beta|,|\alpha-\beta||| \tau(u)-\tau(v)||-2|1-\alpha-\beta|\} .
$$

The desired estimate follows.

This proof shows that there exists an embedding $\varphi_{0}: M G \rightarrow W$ such that $\operatorname{Lip}\left(\varphi_{0}\right) \leq 1$ and $\operatorname{Lip}\left(\varphi_{0}^{-1}\right)$ is bounded from above by an absolute constant. The rest of the proof is quite similar to the proof in Ost11d, we only replace the embedding $\varphi_{0}: M G \rightarrow X$ constructed in [Ost11d by the embedding $\varphi_{0}: M G \rightarrow W$ constructed in Lemma 2.11. For convenience of the reader we reproduce this part of the proof with necessary modifications. We number vertices along short paths using numbers from 1 to $e(G)$ in such a way that vertices numbered 1 correspond to the same long path in the correspondence described above.

At this point we are ready to describe the action of the map $\varphi$ on vertices of short paths. We construct the map $\varphi$ as a map into the Banach space $W \oplus_{1} \mathbb{R}$. This is enough because $W \oplus_{1} \mathbb{R}$ admits a linear bilipschitz embedding into $Z$ with distortion bounded by an absolute constant.

For vertex $w$ of $H$ having number $i$ on the short path $p_{u}$ the image of $w$ in $W \oplus_{1} \mathbb{R}$ is $\varphi(w)=\varphi_{0}(u) \oplus i$ (here we use the same notation $u$ both for a vertex of $G$ and the corresponding vertex in $M G)$.

To map vertices of long paths of $H$ into $W \oplus_{1} \mathbb{R}$ we observe that the numbering of vertices of short paths leads to a one-to-one correspondence between long paths and numbers $\{1, \ldots, e(G)\}$. We define the map $\varphi$ on a long path corresponding to $i$ by $\varphi(w)=\varphi_{0}\left(w^{\prime}\right) \oplus i$, where $w^{\prime}$ is the uniquely determined vertex in a long path of $M G$ corresponding to a vertex $w$ in a long path of $H$.

The fact that $\operatorname{Lip}(\varphi) \leq 1$ follows immediately from the easily verified claim that the distance between $\varphi$-images of adjacent vertices of $H$ is at most 1 (here we use $\left.\operatorname{Lip}\left(\varphi_{0}\right) \leq 1\right)$.

We turn to an estimate of $\operatorname{Lip}\left(\varphi^{-1}\right)$. In this part of the proof we assume that $M>2 e(G)$. Let $w$ and $z$ be two vertices of $H$. As we have already mentioned our 
construction implies that there are uniquely determined corresponding vertices $w^{\prime}$ and $z^{\prime}$ in $M G$.

Obviously there are two possibilities:

(1) $d_{M G}\left(w^{\prime}, z^{\prime}\right) \geq \frac{1}{2} d_{H}(w, z)$. In this case we observe that the definitions of $\varphi$ and of the norm on $W \oplus_{1} \mathbb{R}$ imply that

$$
\|\varphi(w)-\varphi(z)\| \geq\left\|\varphi_{0}\left(w^{\prime}\right)-\varphi_{0}\left(z^{\prime}\right)\right\| \geq d_{M G}\left(w^{\prime}, z^{\prime}\right) / \operatorname{Lip}\left(\varphi_{0}^{-1}\right) \geq \frac{1}{2} d_{H}(w, z) / \operatorname{Lip}\left(\varphi_{0}^{-1}\right) .
$$

(2) $d_{M G}\left(w^{\prime}, z^{\prime}\right)<\frac{1}{2} d_{H}(w, z)$. This inequality implies that there is a path joining $w$ and $z$ for which the naturally defined short-paths-portion is longer than the longpaths-portion. The inequality $M>2 e(G)$ implies that the short-paths-portion of this path consists of one path of length $>\frac{1}{2} d_{H}(w, z)$. This implies that the difference between the second coordinates of $w$ and $z$ in the decomposition $W \oplus_{1} \mathbb{R}$ is > $\frac{1}{2} d_{H}(w, z)$. Thus $\|\varphi(w)-\varphi(z)\|>\frac{1}{2} d_{H}(w, z)$.

Since $\operatorname{Lip}\left(\varphi_{0}^{-1}\right) \geq 1$ (this follows from the assumption $\operatorname{Lip}\left(\varphi_{0}\right) \leq 1$ ), we get $\operatorname{Lip}\left(\varphi^{-1}\right) \leq 2 \operatorname{Lip}\left(\varphi_{0}^{-1}\right)$ in each of the cases (1) and (2). The proof of Theorem 2.1 is completed.

\section{Metric characterization with one test-space}

Bourgain [Bou86] proved that a Banach space is nonsuperreflexive if and only if it admits uniformly bilipschitz embeddings of binary trees of all finite depths (see BL00, pp. 412, 436] for the definition and equivalent characterizations of superreflexivity). Baudier [Bau07] strengthened the "only if" part of this result by proving that each nonsuperreflexive Banach space admits a bilipschitz embedding of an infinite binary tree.

Our purpose in this section is to find similar one-test-space-characterizations for classes of Banach spaces defined in terms of excluded finite-dimensional subspaces. At this moment we do not know how to do this for an arbitrary sequence of finite-dimensional subspaces, we found such a characterization only for increasing sequences of finite-dimensional subspaces.

Theorem 3.1. Let $\left\{X_{n}\right\}_{n=1}^{\infty}$ be an increasing sequence of finite-dimensional Banach spaces with dimensions going to $\infty$. Then there exists an infinite graph $G$ such that the following conditions are equivalent:

- $G$ admits a bilipschitz embedding into a Banach space $X$.

- The spaces $\left\{X_{n}\right\}$ admit uniformly isomorphic embeddings into $X$.

Remark 3.2. Theorem 3.1 is obviously weaker that Theorem 4.1 proved in Section 4. We give an independent proof of Theorem 3.1 because it is substantially simpler. 
Proof of Theorem 3.1. Our proof is based on the construction of graphs providing approximate descriptions of convex sets, see Definition 2.5. We use the following immediate consequence of Theorem 2.8 .

Lemma 3.3. Let $X$ be a finite-dimensional Banach space, $Y$ be a Banach space admitting uniformly bilipschitz embeddings of some $\left(B_{X}(n), 1\right)$-graphs, and $C \in[1, \infty)$ be an upper bound for distortions of these embeddings. Then for each $\varepsilon>0$ there is a linear embedding $T: X \rightarrow Y$ satisfying $\|T\| \cdot\left\|T^{-1}\right\| \leq 3(1+\varepsilon) C$.

Let $L$ be the inductive limit of the sequence $\left\{X_{n}\right\}$, that is, $L=\bigcup_{n=1}^{\infty} X_{n}$ with its natural vector operations and the norm whose restriction to each of $X_{n}$ is the norm of $X_{n}$. So $L$ is an incomplete normed space (we can, of course, consider its completion, but for our purposes completeness is not needed). We construct $G$ as a graph whose vertex set $V$ is a countable infinite subset of $L \oplus_{1} \mathbb{R}$. (We fix the $\ell_{1}$-sum because it is convenient to have a precise formula for the norm, of course in the bilipschitz category all direct sums are equivalent.) The main features of the construction are:

(1) The graph $G$ with its shortest path metric is a locally finite metric space. (Recall that a metric space is called locally finite if all balls of finite radius in it have finite cardinality.)

(2) We have $V=\bigcup_{n=1}^{\infty} V_{n}$, where $V_{n}$ are finite and there exist uniformly bilipschitz embeddings $f_{n}: V_{n} \rightarrow\left(X_{n} \oplus_{1} \mathbb{R}\right)$, where we assume that the distance in $V_{n}$ is inherited from $G$.

(3) The set $V$ endowed with its shortest path metric $d_{G}$ contains images of bilipschitz embeddings of some $\left(B_{X_{n}}(m), 1,2\right)$-graphs (with their shortest path metrics), $m, n \in \mathbb{N}$, with uniformly bounded distortions.

First let us explain why such graph $G$ satisfies the conclusion of Theorem 3.1. Suppose that $X$ is such that the spaces $\left\{X_{n}\right\}$ admit uniformly isomorphic embeddings into $X$. Then condition (2) implies that $V_{n}$ admit uniformly bilipschitz embeddings into $X$. Since $G$ is locally finite, by the main result of Ost11c, we get that $G$ admits a bilipschitz embedding into $X$.

Now suppose that $G$ admits a bilipschitz embedding into $X$. By (3) we get that $X$ admits uniformly bilipschitz embeddings of some $\left(B_{X_{n}}(m), 1\right)$-graphs, $m, n \in \mathbb{N}$. Applying Lemma 3.3, we get that the spaces $\left\{X_{n}\right\}$ admit uniformly isomorphic embeddings into $X$.

We construct $V$ as an infinite union $\bigcup_{n=1}^{\infty} V_{n}$, where each $V_{n}$ is a finite subset of

$$
C_{n}:=\operatorname{conv}\left(\bigcup_{k=1}^{n}\left(B_{X_{k}}(k), s_{k}\right)\right) \subset L \oplus_{1} \mathbb{R},
$$

where $s_{k} \in \mathbb{R}$, the pairs $\left(z, s_{k}\right)$ are in the sense of the decomposition $L \oplus_{1} \mathbb{R}$, and $\left(B_{X_{k}}(k), s_{k}\right)=\left\{\left(z, s_{k}\right): z \in B_{X_{k}}(k)\right\}$. Recall that $B_{X_{k}}(k)$ is the centered at 0 ball of $X_{k}$ of radius $k$. 
Now we describe our choice of $V_{n}$ and $s_{n}$ such that the conditions (1)-(3) above are satisfied. One of the requirements is

$$
s_{n+1}-s_{n}>1 .
$$

We let $s_{1}=0$ and let $V_{1}$ to be a 1 -separated 1-dense subset of $\left(B_{X_{1}}(1), s_{1}\right)$ (see Definition [2.31).

The choice of $s_{2}$ is less restrictive than further choices. We let $s_{2}=2$ and let $V_{2}$ be the extension of $V_{1}$ to a 1-separated 1-dense subset of

$$
C_{2}:=\operatorname{conv}\left(\bigcup_{n=1}^{2}\left(B_{X_{n}}(n), s_{n}\right)\right)
$$

satisfying the condition: some part of $V_{2}$ is a 1-dense in $\left(B_{X_{1}}(2), s_{2}\right)$, and some part of it is a 1-dense in $\left(B_{X_{2}}(2), s_{2}\right)$ (we use $s_{2}-s_{1}>1$ ).

We use the following notation for a subsets $A$ of $L \oplus_{1} \mathbb{R}$ :

$$
A[a, b]:=A \cap\left(L \oplus_{1}[a, b]\right),
$$

where $[a, b]$ is an interval in $\mathbb{R}$. We use similar notation for open and half-open intervals.

Now we turn to the choice of $s_{3}$. We choose $s_{3}$ to satisfy (4) and to be so large that $V_{2}$ is $\left(1+\frac{1}{2}\right)$-dense in $C_{3}\left[s_{1}, s_{2}\right]$. It is easy to see that sufficiently large $s_{3}$ satisfy these conditions. Then we extend $V_{2}$ to a 1 -separated $\left(1+\frac{1}{2}\right)$-dense in $C_{3}$ subset $V_{3}$ in such a way that

- $\left(V_{3} \backslash V_{2}\right) \subset C_{3}\left(s_{2}, s_{3}\right]$

- Some parts of $V_{3}$ are $\left(1+\frac{1}{2}\right)$-dense in the sets $\left(B_{X_{1}}(3), s_{3}\right),\left(B_{X_{2}}(3), s_{3}\right)$, and $\left(B_{X_{3}}(3), s_{3}\right)$, respectively (here we use (44)).

We continue in the following way. We pick $s_{4}$ in such a way that

- $V_{2}$ is $\left(1+\frac{1}{2}+\frac{1}{4}\right)$-dense in $C_{4}\left[s_{1}, s_{2}\right]$.

- $V_{3}$ is $\left(1+\frac{1}{2}+\frac{1}{4}\right)$-dense in $C_{4}\left[s_{1}, s_{3}\right]$.

Now we extend $V_{3}$ to a 1 -separated $\left(1+\frac{1}{2}+\frac{1}{4}\right)$-dense subset $V_{4}$ of $C_{4}$ in such a way that

- $\left(V_{4} \backslash V_{3}\right) \subset C_{4}\left(s_{3}, s_{4}\right]$

- Some parts of $V_{4}$ are $\left(1+\frac{1}{2}+\frac{1}{4}\right)$-dense subsets in $\left(B_{X_{1}}(4), s_{4}\right),\left(B_{X_{2}}(4), s_{4}\right)$, $\left(B_{X_{3}}(4), s_{4}\right)$, and $\left(B_{X_{4}}(4), s_{4}\right)$, respectively (here we use (44)). 
We continue in an obvious way: In step $n$ we pick $s_{n}, s_{n}-s_{n-1}>1$, in such a way that for each $m=2, \ldots, n-1$ we have:

$V_{m}$ is a $\left(1+\frac{1}{2}+\frac{1}{4}+\cdots+\left(\frac{1}{2}\right)^{n-2}\right)$-dense subset of $C_{n}\left[s_{1}, s_{m}\right]$.

We extend $V_{n-1}$ to a 1 -separated $\left(1+\frac{1}{2}+\cdots+\left(\frac{1}{2}\right)^{n-2}\right)$-dense subset $V_{n}$ of $C_{n}$ in such a way that

- $\left(V_{n} \backslash V_{n-1}\right) \subset C_{n}\left(s_{n-1}, s_{n}\right]$

- Some parts of $V_{n}$ are $\left(1+\frac{1}{2}+\cdots+\left(\frac{1}{2}\right)^{n-2}\right)$-dense subsets in

$$
\left(B_{X_{1}}(n), s_{n}\right), \ldots,\left(B_{X_{n}}(n), s_{n}\right),
$$

respectively (here we use (44)).

Let $V=\bigcup_{n=1}^{\infty} V_{n}$ and

$$
C=\operatorname{conv}\left(\bigcup_{n=1}^{\infty}\left(B_{X_{n}}(n), s_{n}\right)\right) \subset L \oplus_{1} \mathbb{R} .
$$

Our construction implies that $V$ is a 1-separated 2-dense subset of $C$. We let $G$ be the corresponding $(C, 1,2)$-graph (see Definition 2.5). It remains to verify that $G$ satisfies the conditions (1)-(3) above.

Condition (1). The set $V$ is a locally finite subset of $L \oplus_{1} \mathbb{R}$ because it is contained in $L \oplus_{1}\left[s_{1}, \infty\right)$ and its intersection with each subset of the form $L \oplus_{1}\left[s_{1}, s_{n}\right]$ is a finite set $V_{n}$. The graph $G$ is locally finite because, by Lemma 2.6, its natural embedding into $L \oplus_{1} \mathbb{R}$ is bilipschitz.

Condition (2). We apply Lemma 2.6 to $V$, the corresponding $(C, 1,2)$-graph, and $L \oplus \mathbb{R}$. We get that the natural embedding of $V$ with the metric inherited from $G$ into $L \oplus \mathbb{R}$ is bilipschitz. Hence its restrictions to $V_{n}$ are uniformly bilipschitz. The fact that the restriction of this map to $V_{n}$ maps $V_{n}$ into $X_{n} \oplus \mathbb{R}$ follows from the definitions.

Condition (3). Our construction of set $V$ is such that it contains subsets which are 1-separated 2-dense subsets in shifted $B_{X_{m}}(n)$. We apply Lemma 2.6 twice. First time to $V$, the corresponding $(C, 1,2)$-graph, and $L \oplus \mathbb{R}$. Second time we apply it to the set $\left(B_{X_{m}}(n), s_{n}\right)$, the corresponding $\left(\left(B_{X_{m}}(n), s_{n}\right), 1,2\right)$-graph (vertex set of this graph is the intersection of $V$ with $\left.\left(B_{X_{m}}(n), s_{n}\right)\right)$. We get that embeddings of all of these graphs into $L \oplus_{1} \mathbb{R}$ have uniformly bounded distortions. Therefore the metrics of these $\left(\left(B_{X_{m}}(n), s_{n}\right), 1,2\right)$-graphs are bilipschitz equivalent to the metrics inherited from $G$. Hence the condition (3) is also satisfied. 


\section{Characterization in terms of an infinite graph with max- imum degree 3}

Our next purpose is to show that the test-space for Theorem 3.1 can be chosen to have maximum degree 3 :

Theorem 4.1. Let $\left\{X_{n}\right\}_{n=1}^{\infty}$ be an increasing sequence of finite-dimensional Banach spaces with dimensions going to $\infty$. Then there exists an infinite graph $H$ with maximum degree 3 such that the following conditions are equivalent:

- $H$ admits a bilipschitz embedding into a Banach space $X$.

- The spaces $\left\{X_{n}\right\}$ admit uniformly isomorphic embeddings into $X$.

Proof. Our proof uses some of the ideas of the proof of Theorem 3.1. For this reason we keep the same notation for some of the objects, although now they are somewhat different. We use Definition 2.3 and the notation introduced in formula (5).

We may assume without loss of generality that $\operatorname{dim} X_{n}=n$. We introduce convex sets $C_{n}$ and $C$ in $L \oplus \mathbb{R}$ of the form

$$
C_{n}:=\operatorname{conv}\left(\bigcup_{k=1}^{n}\left(B_{X_{i(k)}}\left(4^{k}\right), s_{k}\right)\right) \text { and } C=\bigcup_{n=1}^{\infty} C_{n}
$$

where $\{i(k)\}_{k=1}^{\infty}$ is a sequence of natural numbers satisfying $i(1)=1, i(k) \leq i(k+$ $1) \leq i(k)+1$, and such that the equality $i(k+1)=i(k)+1$ holds rarely (the exact condition will be described later), so the dimension of the sets $C_{n}$ increases slowly.

Let $\left\{s_{n}\right\}_{n=1}^{\infty}$ be a sequence of real numbers, such that $s_{1}=0$ and the following two conditions are satisfied:

\section{Gap condition:}

$$
s_{n}-s_{n-1}>6^{n+1} \text {. }
$$

\section{Density condition:}

$$
C_{n-1}\left[a, s_{n-1}\right] \text { is }\left(\frac{1}{2}\right)^{n-2}-\text { dense in } C_{n}\left[a, s_{n-1}\right] \text { for every } a \in\left[s_{1}, s_{n-1}\right] \text {. }
$$

Remark 4.2. It is easy to verify that condition (7) is satisfied for each sufficiently rapidly increasing sequence $\left\{s_{n}\right\}_{n=1}^{\infty}$.

We construct two sequences of finite subsets in $L \oplus \mathbb{R},\left\{A_{n}\right\}_{n=1}^{\infty}$ and $\left\{B_{n}\right\}_{n=2}^{\infty}$. The desired properties of these sequences of sets are the following:

1. $A_{n}$ is $2^{n}$-separated $2^{n}$-dense subset in $C_{n}\left[s_{n-1}+2^{n}, s_{n}\right]$. If $n=1$, this condition is replaced by: $A_{1}$ is a 2 -separated 2 -dense set in $C_{1}$.

2. $A_{n}$ contains $2^{n}$-separated $2^{n}$-dense subsets in $\left\{\left(B_{X_{i(k)}}\left(4^{n}\right), s_{n}\right)\right\}_{k=1}^{n}$. 
3. $B_{n}, n \geq 2$ is a $2^{n}$-separated subset of $C_{n}\left(s_{n-1}, s_{n-1}+2^{n}\right)$ such $d\left(A_{n}, B_{n}\right) \geq 2^{n}$, $d\left(A_{n-1}, B_{n}\right) \geq 2^{n}$ and $A_{n-1} \cup B_{n} \cup A_{n}$ is a $2^{n-1}$-separated $2^{n}$-dense subset in $C_{n}\left[s_{n-2}+2^{n-1}, s_{n}\right]$. If $n=2$, the last condition is replaced by: 2 -separated and $2^{2}$-dense in $C_{2}\left[s_{1}, s_{2}\right]=C_{2}$.

We construct such sets in steps. First we construct $A_{n}$, then $B_{n}$ (for $n \geq 2$ ). We start by letting $A_{1}$ to be any 2-separated 2-dense subset of $\left(B_{X_{1}}(4), s_{1}\right)$.

The construction of $A_{n}(n \geq 2)$ starts with picking a $2^{n}$-separated $2^{n}$-dense subset of

$$
\left(B_{X_{1}}\left(4^{n}\right), s_{n}\right) \text {. }
$$

Then we gradually extend this subset to $2^{n}$-separated $2^{n}$-dense subsets of

$$
\left(B_{X_{i(2)}}\left(4^{n}\right), s_{n}\right), \ldots\left(B_{X_{i(n)}}\left(4^{n}\right), s_{n}\right)
$$

Observe that our description of the sequence $\{i(n)\}$ implies that many sets in the sequence (8) are the same.

We complete the construction of $A_{n}$ extending the obtained set to a $2^{n}$-separated $2^{n}$-dense subset of $C_{n}\left[s_{n-1}+2^{n}, s_{n}\right]$.

To construct $B_{n}$ we remove from $C_{n}\left(s_{n-1}, s_{n-1}+2^{n}\right)$ all elements which are covered by $2^{n}$-balls centered in $A_{n-1} \cup A_{n}$. If the obtained set $R$ is empty, we let $B_{n}=\emptyset$. Otherwise we let $B_{n}$ be a $2^{n}$-separated $2^{n}$-dense subset of $R$.

The only condition which has to be verified is the condition that $A_{n-1} \cup B_{n} \cup A_{n}$ is $2^{n}$-dense in $C_{n}\left[s_{n-2}+2^{n-1}, s_{n}\right]$ (and its version for $n=2$ ). Here we use the condition (77). By this condition, since $A_{n-1}$ is $2^{n-1}$-dense in $C_{n-1}\left[s_{n-2}+2^{n-1}, s_{n-1}\right]$, it is $\left(2^{n-1}+\left(\frac{1}{2}\right)^{n-2}\right)$-dense in $C_{n}\left[s_{n-2}+2^{n-1}, s_{n-1}\right]$. Since $\left(2^{n-1}+\left(\frac{1}{2}\right)^{n-2}\right)<2^{n}$, the conclusion follows from the construction of $A_{n}$ and $B_{n}$.

Let

$$
V=\left(\bigcup_{n=1}^{\infty} A_{n}\right) \cup\left(\bigcup_{n=2}^{\infty} B_{n}\right)
$$

We create a weighted graph with the vertex set $V$ by joining a vertex $v \in\left(A_{n} \cup\right.$ $\left.B_{n}\right), n \geq 2$, to all vertices of

$$
\left(\bigcup_{k=1}^{n} A_{k}\right) \cup\left(\bigcup_{k=2}^{n} B_{k}\right)
$$

which are within distance $3 \cdot 2^{n}$ to $v$ in the normed space $L \oplus_{1} \mathbb{R}$. (Also we join each vertex $v \in A_{1}$ to all vertices of $A_{1}$ which are within distance 6 to $v$.) The inequality (6) implies that in this way vertices of $A_{n} \cup B_{n}$ are joined only to some of the vertices in $A_{n-1} \cup B_{n-1}, A_{n} \cup B_{n}$, and $A_{n+1} \cup B_{n+1}$. The vertex $v$ is joined to some vertices in $A_{n+1} \cup B_{n+1}$ if $v$ is within distance $3 \cdot 2^{n+1}$ in $L \oplus_{1} \mathbb{R}$ to those vertices. 
We assign weight (length) $2^{n}$ to all edges joining $v \in\left(A_{n} \cup B_{n}\right)$ with vertices of $\left(A_{n-1} \cup B_{n-1}\right) \cup\left(A_{n} \cup B_{n}\right)$. Thus we assign weight (length) $2^{n+1}$ to the edges joining $v \in\left(A_{n} \cup B_{n}\right)$ with vertices of $\left(A_{n+1} \cup B_{n+1}\right)$. It is clear that we get a well-defined weighted graph. We denote the obtained weighted graph by $W$ and endow it with its (weighted) shortest path metric.

We estimate the number of edges incident to $v \in\left(A_{n} \cup B_{n}\right)$ in the following way. All vertices joined to $v$ by edges are in a ball of radius $3 \cdot 2^{n+1}$ centered at $v$. The distance between two vertices joined to $v$ is at least $2^{n-1}$ because all such vertices are in the set $A_{n-1} \cup B_{n-1} \cup A_{n} \cup B_{n} \cup A_{n+1} \cup B_{n+1}$, and it is clear from the conditions on $A_{n}$ and $B_{n}$ that the set $A_{n-1} \cup B_{n-1} \cup A_{n} \cup B_{n} \cup A_{n+1} \cup B_{n+1}$ is $2^{n-1}$-separated. All of the elements of this set are in $X_{i(n+1)} \oplus \mathbb{R}$, and the dimension of this space is $d(n)=i(n+1)+1$. Therefore $X_{i(n+1)} \oplus \mathbb{R}$-balls of radiuses $2^{n-2}$ centered at points joined to $v$ with an edge have disjoint interiors and are contained in a ball of radius $3 \cdot 2^{n+1}+2^{n-2}$ centered at $v$. Comparing the volumes of the union of the $2^{n-2}$ balls and the $\left(3 \cdot 2^{n+1}+2^{n-2}\right)$-ball containing them (it is the standard volumetric argument, see e.g. [MS86, Lemma 2.6]), we get that the number of vertices adjacent to $v$ is at most

$$
\left(\frac{3 \cdot 2^{n+1}+2^{n-2}}{2^{n-2}}\right)^{d(n)}=25^{d(n)} .
$$

Lemma 4.3. The natural embedding of $W$ into the normed space $L \oplus_{1} \mathbb{R}$ has distortion $\leq 3$. More precisely, its Lipschitz constant is $\leq 3$, and the Lipschitz constant of the inverse map is $\leq 1$.

Proof. The statement about the Lipschitz constant of the natural embedding is immediate. In fact, ends of an edge of length $2^{n}$ in $W$ correspond to a vector in $L \oplus_{1} \mathbb{R}$ whose length is $\leq 3 \cdot 2^{n}$.

The fact that the Lipschitz constant of the inverse map is $\leq 1$ can be proved as follows:

Let $a$ and $b$ be vertices of $W$. We may assume that $b \in\left(A_{n} \cup B_{n}\right)$ and $a \in\left(A_{k} \cup B_{k}\right)$ for some $k \leq n$. We use double induction. This means the following: First we prove the result for $n=1$ using induction on $\lfloor|| a-b \| / 2\rfloor$ (here we use Lemma 2.6).

Next, we assume that the result holds for $n=m$ and any $k \leq m$. We show that this assumption can be used to prove the result for $n=m+1$ using the induction on $\left\lfloor|| a-b \| /\left(2^{m+1}\right)\right\rfloor$.

So let us follow the described program. If we divide all distances by 2 , the desired inequality for $a, b \in A_{1}$ is a special case of Lemma 2.6 for $\alpha=\beta=1$.

Assumption: Now we assume that we have proved the statement for all pairs $b \in\left(A_{m} \cup B_{m}\right), a \in\left(A_{k} \cup B_{k}\right), k \leq m$.

We show that this assumption can be used to prove the statement for $b \in\left(A_{m+1} \cup\right.$ $\left.B_{m+1}\right), a \in\left(A_{k} \cup B_{k}\right), k \leq m+1$, using induction on $\left\lfloor|| a-b \| /\left(2^{m+1}\right)\right\rfloor$. 
If $\left\lfloor\|a-b\| /\left(2^{m+1}\right)\right\rfloor \leq 2$, then $a$ and $b$ are joined by an edge of length $2^{m+1}$. In addition, $\|a-b\| \geq 2^{m+1}$ (see the conditions 1 and 3 in the description of $A_{n}$ and $\left.B_{n}\right)$. So in this case we get the desired $d_{W}(a, b) \leq\|a-b\|$.

Induction Hypothesis: Suppose that the statement has been proved for all pairs $b \in\left(A_{m+1} \cup B_{m+1}\right), a \in\left(A_{k} \cup B_{k}\right), k \leq m+1$, and

$$
\left\lfloor\|a-b\| /\left(2^{m+1}\right)\right\rfloor \leq D .
$$

We show that this implies the same conclusion for pairs $b \in\left(A_{m+1} \cup B_{m+1}\right), a \in$ $\left(A_{k} \cup B_{k}\right), k \leq m+1$, satisfying $\left\lfloor\|a-b\| /\left(2^{m+1}\right)\right\rfloor \leq D+1$.

We need to consider the case where the inequality $(D+2) \cdot 2^{m+1}>\|a-b\| \geq$ $(D+1) \cdot 2^{m+1}$ is satisfied, $D \geq 2, D \in \mathbb{N}$. In such a case let $\widetilde{b}$ be the vector on the line segment joining $b$ to $a$ at distance $2 \cdot 2^{m+1}$ from $b$.

It is clear from the construction that $\tilde{b} \in C_{m+1}$. Therefore (see condition 3 in the list of conditions on $A_{n}$ and $\left.B_{n}\right)$ there is a point $\widehat{b} \in V$ such that $\|\widehat{b}-\widetilde{b}\| \leq 2^{m+1}$. We have $\|\widehat{b}-b\| \leq 3 \cdot 2^{m+1}$ and

$$
\|\widehat{b}-a\| \leq\|b-a\|-2^{m+1} .
$$

Also we have $\widehat{b} \in A_{m} \cup B_{m} \cup A_{m+1} \cup B_{m+1}$. Therefore $d_{W}(\widehat{b}, b) \leq 2^{m+1}$.

In the case when $\widehat{b} \in A_{m+1} \cup B_{m+1}$, we get the desired conclusion using the Induction Hypothesis as follows: The inequality (10) implies that the pair $a, \widehat{b}$ satisfies the inequality (9). By the Induction Hypothesis,

$$
\|a-\widehat{b}\| \geq d_{W}(a, \widehat{b}) .
$$

Therefore

$$
\|a-b\| \geq 2^{m+1}+\|a-\widehat{b}\| \geq 2^{m+1}+d_{W}(a, \widehat{b}) \geq d_{W}(a, b) .
$$

It remains to consider the case $\widehat{b} \in A_{m} \cup B_{m}$. This case is to be divided into two subcases: $k=m+1$ and $k \leq m$. In the latter case we use the assumption that we have proved the statement for points in $\left(\bigcup_{i=1}^{m} A_{i}\right) \cup\left(\bigcup_{i=2}^{m} B_{i}\right)$. In the former case we use the Induction Hypothesis.

The graph $W$ contains $\left(B_{X_{k}}\left(2^{n}\right), 1\right)$-graphs (in the sense of Definition 2.5) as subgraphs for all $k, n \in \mathbb{N}$, with all edges having the same weight of $2^{n}$. This follows from the choice of $4^{n}$ as the diameter of the ball used in the construction of $C_{n}$ and the fact that $A_{n}$ contains subsets which are $2^{n}$-separated and $2^{n}$-dense in the ball $\left(B\left(X_{k}\left(4^{n}\right)\right), s_{n}\right)$ for $k \leq i(n)$ (by combining these facts with our definitions). We claim that these $\left(B\left(X_{k}\left(2^{n}\right)\right), 1\right)$-graphs embed in a uniformly bilipschitz way into $W$. This claim can be proved in the following way: Applying Lemma 2.6 we get that the natural embeddings of the $\left(B\left(X_{k}\left(4^{n}\right)\right), 2^{n}\right)$-graphs (constructed using the $2^{n}$-separated and $2^{n}$-dense in the ball $\left.\left(B\left(X_{k}\left(4^{n}\right)\right), s_{n}\right)\right)$ into $L \oplus_{1} \mathbb{R}$ are uniformly 
bilipschitz. By Lemma 4.3, the natural embedding of $W$ into $L \oplus_{1} \mathbb{R}$ is also bilipschitz. The conclusion on uniformity of bilipschitz embeddings of the $\left(B\left(X_{k}\left(2^{n}\right)\right), 1\right)$ graphs into $W$ follows. Thus, by Lemma 2.7, bilipschitz embeddability of the graph $W$ into a Banach space $X$ implies that $X$ admits uniformly isomorphic embeddings of the spaces $\left\{X_{i}\right\}_{i=1}^{\infty}$.

Now we construct an unweighted graph $H$ with maximum degree 3 whose existence is claimed in Theorem 4.1. The graph $H$ will admit a bilipschitz embedding into $\left(L \oplus_{1} \mathbb{R}\right) \oplus_{1} \mathbb{R}$. The graph $H$ will be a modification of the weighted graph $W$. As in the construction of Section 2, the graph $H$ consists of long paths and short paths. Short paths correspond to vertices of $W$, long paths correspond to (weighted) edges of $W$. The length of a short path corresponding to a vertex in $A_{n} \cup B_{n}$ is $2 \cdot 25^{d(n+1)}$. These lengths of short paths are chosen because they provide a sufficient number of colors for the coloring introduced in the next paragraph.

We are going to color edges of $W$. For our purposes we need a proper edge coloring (that is, edges having a common end should have different colors). Of course, since the degrees of $W$ are unbounded, we need infinitely many colors. Our purpose is to bound from above the number of colors used for edges incident with a vertex $v \in A_{n} \cup B_{n}$ by $2 \cdot 25^{d(n+1)}$. To achieve this goal we order vertices in $V$ according to the magnitude of their $\mathbb{R}$-coordinate in the decomposition $L \oplus \mathbb{R}$ (starting with those for which the $\mathbb{R}$-coordinate is 0 ), resolving ties in an arbitrary way.

We color edges incident with the first vertex arbitrarily (there are at most $25^{d(1)}$ of such edges). For each of the further vertices in our list we need to color all uncolored edges incident with them. We do this according to the following procedure. Let $v \in A_{n} \cup B_{n}$ be the next vertex in our list. We pick an uncolored edge incident with $v$, let $u$ be the other end of this edge. We cannot use for the edge $v u$ the colors which have already been used for other edges incident to $v$ and to $u$. There are at most $25^{d(n)}-1$ colors already used for edges incident with $v$. As for $u$, we know that (see the construction of $W$ ) $u \in A_{n-1} \cup B_{n-1} \cup A_{n} \cup B_{n} \cup A_{n+1} \cup B_{n+1}$, therefore the degree of $u$ is $\leq 25^{d(n+1)}$. Therefore among $2 \cdot 25^{d(n+1)}$ colors there should be an available color for the edge $v u$.

Now we create a graph as in Section 2. The only difference is that we paste a long path to the level corresponding to its color (rather than to the level corresponding to the path itself as we did in Section 2). The length of the long path corresponding to an edge of $W$ of weight $2^{n}$ is $M \cdot 2^{n}$, where $M$ is a positive integer which we are going to select now, together with the sequence $\{i(n)\}_{n=1}^{\infty}$ (which, as we have already mentioned, is a slowly increasing sequence). The main condition describing our choice of both objects is

$$
M \cdot 2^{n}>4 \cdot 25^{d(n+1)} .
$$

(Recall that $d(n)=i(n+1)+1$.) This condition ensures that the length of a long path is larger than the sum of the lengths of the short paths at its ends. 
We get an unweighted graph, let us denote it $H$. The maximum degree of $H$ is 3 because we use a proper edge coloring. The graph $W$ admits a bilipschitz embedding into $H$ : consider the map which maps each vertex of $W$ to the vertex of level 1 on the corresponding short path. The Lipschitz constant of this embedding is $\leq 2 \cdot M$ by (11). The Lipschitz constant of the inverse map is $\leq M^{-1}$. In fact, if we consider two vertices in the image of $W$, and join them by a shortest path in $H$, the path goes through some collection of short paths (possibly at the end vertices only). The vertices in $W$ corresponding to these short paths form a path in $W$. Each time the weight of the edge in $W$ is $M^{-1} \times($ the length of the corresponding path in $H$ ). The conclusion about the Lipschitz constant of the inverse map follows.

Observe that the graph $H$ is locally finite because its maximum degree is 3 . It remains to show that the graph $H$ admits a bilipschitz embedding into any Banach space $X$ containing uniformly isomorphic $\left\{X_{i}\right\}$. We do this by proving the fact that the graph $H$ admits a bilipschitz embedding into $\left(L \oplus_{1} \mathbb{R}\right) \oplus_{1} \mathbb{R}$. By the finite determination result of Ost11c this is enough because it is easy to see that finite dimensional subspaces of the space $\left(L \oplus_{1} \mathbb{R}\right) \oplus_{1} \mathbb{R}$ are uniformly isomorphic to subspaces in $\left\{X_{i}\right\}$.

Lemma 4.4. The graph $H$ admits a bilipschitz embedding into $\left(L \oplus_{1} \mathbb{R}\right) \oplus_{1} \mathbb{R}$.

Proof. Vertices of $W$ are in $L \oplus_{1} \mathbb{R}$. We map the short path corresponding to a vertex $v \in A_{n} \cup B_{n}$ onto those points of the line segment joining $(M v, 1)$ with $(M v, 2$. $25^{d(n+1)}$ ) whose second coordinate is an integer. (The number $M$ is introduced by (11). In a pair $(M v, a)$ the first component is in $L \oplus_{1} \mathbb{R}$ and the second component is in the second $\mathbb{R}$-summand.)

Now we describe our map for long paths of $H$. For each long path of $H$, or, what is the same, for each edge $u v$ of $W$ we pick a vector $x_{u v} \in L$ (the space $L$ is identified with the corresponding summand in $\left.\left(L \oplus_{1} \mathbb{R}\right) \oplus_{1} \mathbb{R}\right)$. Suppose that the edge $u v$ has weight $2^{n}$ and color $i$ in the coloring above. We number vertices of the long path corresponding to $u v$ as $u_{0}, u_{1}, \ldots, u_{N}$, where $N=M \cdot 2^{n}, u$ corresponds to $u_{0}$ and $v$ corresponds to $u_{N}$. The image of $u_{m}(m=0,1, \ldots, N)$ under the map which we are constructing is given by

$$
T u_{m}= \begin{cases}\left(\left(1-\frac{m}{N}\right) M u+\frac{m}{N} M v+m x_{u v}, i\right) & \text { if } m \leq \frac{N}{2} \\ \left(\left(1-\frac{m}{N}\right) M u+\frac{m}{N} M v+(N-m) x_{u v}, i\right) & \text { if } m \geq \frac{N}{2}\end{cases}
$$

So we map vertices of the long path in $H$ corresponding to an edge $u v$ of $W$ onto a sequence of evenly distributed points in the union of two line segments joining $(M u, i)$ and $(M v, i)$. We introduce also a map $O$ given by $O u_{m}=\left(1-\frac{m}{N}\right) M u+$ $\frac{m}{N} M v$.

The map $T$ introduced by (12) is a Lipschitz map of the vertex set of $H$ into $\left(L \oplus_{1} \mathbb{R}\right) \oplus_{1} \mathbb{R}$ for an arbitrary uniformly bounded set of vectors $\left\{x_{u v}\right\}$ in $L$. To show this it suffices to find a bound for the distances between images of ends of an edge of $H$. For short-path-edges the distances are equal to 1 because their ends 
are mapped onto pairs of the form $(M v, i),(M v, i+1)$. For a long-path-edge, the distance between the ends is

$$
\left\|\frac{1}{N} M v-\frac{1}{N} M u \pm x_{u v}\right\|
$$

This norm can be estimated from above by $3+\sup _{u v}\left\|x_{u v}\right\|$ (we use the estimate for the Lipschitz constant of Lemma 4.3).

Therefore the purpose of a suitable selection of the set $\left\{x_{u v}\right\}$ is to ensure that $T^{-1}$ is Lipschitz. In a similar situation in Section 2 we used Auerbach bases. For this construction we use a somewhat different type of biorthogonal sequences. We use systems whose existence was shown by Ovsepian-Pełczyński OP75. We mean the following result proved in OP75] (see also [LT77, p. 44]):

Theorem 4.5. There is an absolute constant $C>0$ such that for each separable Banach space $Z$, each sequence $\left\{f_{i}^{*}\right\} \subset Z^{*}$, and each sequence $\left\{f_{i}\right\} \subset Z$ there exists a biorthogonal sequence $\left\{z_{i}, z_{i}^{*}\right\}_{i=1}^{\infty}$ in $Z$ such that $\left\|z_{i}\right\| \leq C$, $\left\|z_{i}^{*}\right\| \leq C$, the linear span of $\left\{z_{i}\right\}$ contains the sequence $\left\{f_{i}\right\}$, and the linear span of $\left\{z_{i}^{*}\right\}$ contains the sequence $\left\{f_{i}^{*}\right\}$.

Remark 4.6. Pełczyński [Pel76] and Plichko [Pli76] proved that the constant $C$ can be chosen to be an arbitrary number $>1$.

We apply Theorem 4.5 to $Z=L$ in the following situation. We form the sequence $\left\{f_{i}^{*}\right\}$ in the following way. We denote by $P_{L}: L \oplus \mathbb{R} \rightarrow L$ the natural projection. For any two edges $u v$ and $w z$ in $W$ we consider all vectors of the form

$$
P_{L}\left(O u_{m}-O w_{p}\right)
$$

for all admissible values of $m$ and $p$. The map $O$ was defined after formula (12) and $O w_{p}$ is the image of a vertex $w_{p}$ of a long path corresponding to $w z$.

For each vector of the form (13) we pick a supporting functional $f^{*} \in L^{*}$, that is, a functional $f^{*}$ satisfying

$$
f^{*}\left(P_{L}\left(O u_{m}-O w_{p}\right)\right)=\left\|P_{L}\left(O u_{m}-O w_{p}\right)\right\|
$$

and $\left\|f^{*}\right\|=1$. There are countably many such functionals, so we can form a sequence $\left\{f_{i}^{*}\right\}$ containing all of them. Also we form a sequence $\left\{f_{i}\right\}$ containing all of the vectors of the form (13).

Now we describe a suitable choice of the vectors $x_{u v}$ for (12). We enumerate edges of $W$ in the non-decreasing order of the larger $\mathbb{R}$-coordinates of their ends, resolving ties arbitrarily. Let $u v$ be the first edge in the ordering. We pick as $x_{u v}$ an element of the sequence $\left\{z_{i}\right\}$ satisfying the following two conditions:

- $z_{i}$ is annihilated by all functionals $f_{j}^{*}$ of the sequence $\left\{f_{i}^{*}\right\}$ supporting vectors $P_{L}\left(O u_{m}-O w_{p}\right)$, where $u_{m}$ is in the long path corresponding to $u v$ and $w_{p}$ is in the long path corresponding to an edge $w z$ for which the smaller $\mathbb{R}$-coordinate of its ends is $\leq s_{3}$. 
- $x_{u v}^{*}:=z_{i}^{*}$ annihilates all vectors $f_{j}$ of the sequence $\left\{f_{i}\right\}$ of the form $P_{L}\left(O u_{m}-\right.$ $\left.O w_{p}\right)$, where $u_{m}$ is in the long path corresponding to $u v$ and $w_{p}$ is in the long path corresponding to an edge $w z$ for which the smaller $\mathbb{R}$-coordinate of its ends is $\leq s_{3}$.

Such pair $z_{i}, z_{i}^{*}$ exists because there are finitely many $f_{j}$ and $f_{j}^{*}$ satisfying the conditions. (Here we use the following conditions of Theorem 4.5: the linear span of $\left\{z_{i}\right\}$ contains the sequence $\left\{f_{i}\right\}$, and the linear span of $\left\{z_{i}^{*}\right\}$ contains the sequence $\left\{f_{i}^{*}\right\}$.)

We make a similar choice of $x_{u v}$ for all further edges in the ordering. More details: If we consider an edge for which the larger $\mathbb{R}$-coordinate of an end is in the interval $\left(s_{n-1}, s_{n}\right]$, we replace $s_{3}$ by $s_{n+2}$ in the conditions above. Also we pick different pairs $z_{i}, z_{i}^{*}$ for different edges $u v$.

With this choice of vectors $x_{u v}$, let us estimate from above the Lipschitz constant of the inverse of $T$. Let $x, y$ be two vertices of $H$. Let $x$ be on a long path joining $u$ and $v$ and $y$ be on a long path joining $w$ and $z$ (this is a generic description because $x$ and $y$ are allowed to be the end vertices of the long paths). We need to estimate from above the quotient

$$
\frac{d_{H}(x, y)}{\|T x-T y\|}
$$

We consider a shortest $x y$-path in $H$. It has naturally defined short-path portion and long-path portion. There are two cases: (1) The length of the short-path portion of this path has length $\geq \frac{1}{2} d_{H}(x, y)$; The length of the long-path portion of of this path has length $>\frac{1}{2} d_{H}(x, y)$.

The construction of the graph $H$ (see inequality (11)) is such that in the case (1) the short-path portion consists of just one piece. Let the short path portion start at level (color) $i$ and end at level $j$. Then $|i-j| \geq \frac{1}{2} d_{H}(x, y)$. On the other hand, since the $\operatorname{sum}\left(L \oplus_{1} \mathbb{R}\right) \oplus_{1} \mathbb{R}$ is direct, we have $\|T x-T y\| \geq|i-j| \geq \frac{1}{2} d_{H}(x, y)$.

In the case (2) we ignore the difference in the second $\mathbb{R}$-coordinate (caused by the different colors of the edges $u v$ and $w z$ ). There are two subcases: Subcase (A): The vertices $x$ and $y$ are on the same long path; Subcase (B): The vertices $x$ and $y$ are on different long paths.

Subcase (A): Observe that our construction is such that there is a functional supporting $P_{L}(u-v)$ (let us denote it $f_{u v}^{*}$ ) which annihilates by $x_{u v}$ (because $u-v$ is of the form $\left.O u_{m}-O w_{p}\right)$. Let $x=u_{p}, y=u_{s}$, we have $d_{H}(x, y)=|s-p|$ and

$$
|| T u_{p}-T u_{s}\left\|\geq\left|f_{u v}^{*} P_{L}\left(O u_{p}-O u_{s}\right)\right|=\frac{M|s-p|}{N}|| P_{L}(u-v)\right\| .
$$

If $\left\|P_{L}(u-v)\right\|=\left\|P_{L} u-P_{L} v\right\|$ is a nontrivially large part of $\|u-v\| \geq \frac{N}{M}$, we get the desired estimate. 
If $\left\|P_{L} u-P_{L} v\right\|$ is only a small part of $\|u-v\|$, then the difference between the $\mathbb{R}$-coordinates of $u$ and $v$ is the large part of $\|u-v\|$. Denoting the projection of $L \oplus \mathbb{R}$ to $\mathbb{R}$ by $P_{\mathbb{R}}$, we use $P_{\mathbb{R}}\left(x_{u v}\right)=0$ and get

$$
|| T u_{p}-T u_{s} \| \geq \frac{M|s-p|}{N}\left|P_{\mathbb{R}} u-P_{\mathbb{R}} v\right|,
$$

so we get the estimate in this case, too.

Subcase (B): Let $x$ be on a long path corresponding to an edge $u v$ in $W$, and $y$ be on a long path corresponding to $w z$. Then there are two possibilities:

(i) One of the edges $u v$ and $w z$ has the largest $\mathbb{R}$-coordinate of its ends in the interval $\left(s_{n-1}, s_{n}\right]$, and the other edge has the least $\mathbb{R}$-coordinate of its ends in the interval $\left[s_{m}, s_{m+1}\right)$, where $m \geq n+2$

(ii) It is not the case.

Subsubcase (i): Let $x=u_{m}$ and $y=w_{p}$. Ignoring the second $\mathbb{R}$-coordinate (in the sum $L \oplus \mathbb{R} \oplus \mathbb{R})$ and using $P_{\mathbb{R}} x_{u v}=P_{\mathbb{R}} x_{w z}=0$ we get

$$
|| T x-T y|| \geq M\left|P_{\mathbb{R}} u-P_{\mathbb{R}} w\right| \geq M\left(s_{m}-s_{n}\right),
$$

where $u$ and $w$ are the corresponding vertices picked in such a way that $u$ has larger $\mathbb{R}$-coordinate than $v$ and $w$ has smaller $\mathbb{R}$-coordinate than $w$. On the other hand

$$
\begin{aligned}
d_{H}(x, y) & \leq d_{H}(u, w)+M 2^{n}+M 2^{m+1} \\
& \leq M|| u-w||+M 2^{n}+M 2^{m+1} \\
& \leq M\left|P_{\mathbb{R}} u-P_{\mathbb{R}} w\right|+M 2^{n}+M 2^{m+1}+M 4^{n}+M 4^{m+1} \\
& \leq M\left|P_{\mathbb{R}} u-P_{\mathbb{R}} w\right|+M\left(s_{m}-s_{n}\right) \\
& \leq 2 M\left|P_{\mathbb{R}} u-P_{\mathbb{R}} w\right| .
\end{aligned}
$$

To get these inequalities we use

- The triangle inequality in $H$ for the first inequality.

- Lemma 4.3 for the second inequality.

- The observation that $\left\|P_{L} z\right\| \leq 4^{n}$ if $z \in C_{n}$ for the third inequality (see the definition of $C_{n}$ ).

- The gap condition (6) for the fourth inequality.

- The second inequality in (15) for the fifth inequality.

The conclusion follows.

Subsubcase (ii): We may assume without loss of generality that $x$ is closer (in $H$ ) to the short path corresponding to $u$ than to the short path corresponding to $v$ and 
that $y$ is closer to the short path corresponding to $w$ rather than to the short path corresponding to $z$. We have

$$
\begin{aligned}
\| T x & -T y \| \\
& \geq\left\|\left(1-\frac{m}{N}\right) M u+\frac{m}{N} M v+m x_{u v}-\left(1-\frac{p}{N^{\prime}}\right) M w-\frac{p}{N^{\prime}} M z-p x_{w z}\right\|,
\end{aligned}
$$

where $N^{\prime}$ is the length of the long path corresponding to $w z$. Let us denote the vector whose norm is taken in the right-hand side of (16) by $B$. We get

$$
\begin{gathered}
|| T x-T y \| \geq\left|x_{u v}^{*}(B)\right|=m . \\
|| T x-T y \| \geq\left|x_{w z}^{*}(B)\right|=p .
\end{gathered}
$$

Writing $x_{u v}^{*}(B), x_{w z}^{*}(B)$ we mean that the functionals $x_{u v}^{*}, x_{w z}^{*} \in L^{*}$ act on a vector $t \in L \oplus \mathbb{R}$ by acting on $P_{L} t$. We use the fact that the difference $\left(1-\frac{m}{N}\right) M u+$ $\frac{m}{N} M v-\left(1-\frac{p}{N^{\prime}}\right) M w-\frac{p}{N^{\prime}} M z$ is of the from $O u_{m}-O w_{p}$ for edges satisfying the conditions above. Thus this difference is annihilated by $x_{u v}^{*}$ and $x_{w z}^{*}$.

We apply the triangle inequality to (16) and get

$$
\begin{aligned}
\| T x & -T y \| \\
& \geq M\|u-w\|-\frac{m M}{N}\|u-v\|-\frac{p M}{N^{\prime}}\|w-z\|-m C-p C \\
& =M\|u-w\|-m\left(\frac{M}{N}\|u-v\|+C\right)-p\left(\frac{M}{N^{\prime}}\|w-z\|+C\right) \\
& \geq d_{H}(u, w)-m\left(\frac{M}{N}\|u-v\|+C\right)-p\left(\frac{M}{N^{\prime}}\|w-z\|+C\right),
\end{aligned}
$$

where $C=\sup _{i}\left\|z_{i}\right\|=\sup _{u, v}\left\|x_{u v}\right\|$. (We used Lemma 4.3 to get the last inequality.) Observe that the numbers in brackets in the last line are bounded by an absolute constant, let us denote it by $D$. We also have $d_{H}(x, y) \leq d_{H}(u, w)+m+p$. Therefore

$$
\frac{d_{H}(x, y)}{\|T x-T y\|} \leq \min \left\{\frac{d_{H}(u, w)+m+p}{d_{H}(u, w)-D(m+p)}, \frac{d_{H}(u, w)+m+p}{m}, \frac{d_{H}(u, w)+m+p}{p}\right\} .
$$

It is easy to see that the minimum in the last formula is bounded from above by an absolute constant.

\section{References}

[Aue30] H. Auerbach, On the area of convex curves with conjugate diameters (Polish), Ph. D. thesis, University of Lwów, 1930. 
[Bau07] F. Baudier, Metrical characterization of super-reflexivity and linear type of Banach spaces, Archiv Math., 89 (2007), no. 5, 419-429; arXiv:0704.1955.

[Bau09+] F. Baudier, Embeddings of proper metric spaces into Banach spaces, Houston J. Math., to appear; arXiv:0906.3696.

[Beg99] B. Begun, A remark on almost extensions of Lipschitz functions, Israel J. Math., 109 (1999), 151-155.

[BL00] Y. Benyamini, J. Lindenstrauss, Geometric Nonlinear Functional Analysis, volume 1, American Mathematical Society, Providence, R.I., 2000.

[Bou86] J. Bourgain, The metrical interpretation of superreflexivity in Banach spaces, Israel J. Math., 56 (1986), no. 2, 222-230.

[Bou87] J. Bourgain, Remarks on the extension of Lipschitz maps defined on discrete sets and uniform homeomorphisms, in: Geometrical aspects of functional analysis (1985/86), 157-167, Lecture Notes in Math., 1267, Springer, Berlin, 1987.

[BMW86] J. Bourgain, V. Milman, H. Wolfson, On type of metric spaces, Trans. Amer. Math. Soc., 294 (1986), no. 1, 295-317.

[DS58] N. Dunford, J. T. Schwartz, Linear Operators. Part I: General Theory, New York, Interscience Publishers, 1958.

[GNS11] O. Giladi, A. Naor, G. Schechtman, Bourgain's discretization theorem; arXiv:1110.5368

[Gro93] M. Gromov, Asymptotic invariants of infinite groups, in: A. Niblo, M. Roller (Eds.) Geometric group theory, London Math. Soc. Lecture Notes, 182, 1-295, Cambridge University Press, 1993.

[Han77] Handbook of mathematical logic. Edited by Jon Barwise. With the cooperation of H. J. Keisler, K. Kunen, Y. N. Moschovakis, A. S. Troelstra. Studies in Logic and the Foundations of Mathematics, Vol. 90, North-Holland Publishing Co., Amsterdam-New York-Oxford, 1977.

[HM82] S. Heinrich, P. Mankiewicz, Applications of ultrapowers to the uniform and Lipschitz classification of Banach spaces, Studia Math., 73 (1982), no. 3, 225-251.

[JS09] W. B. Johnson, G. Schechtman, Diamond graphs and super-reflexivity, J. Topol. Anal., 1 (2009), no. $2,177-189$.

[LT77] J. Lindenstrauss, L. Tzafriri, Classical Banach spaces, v. I, Berlin, Springer-Verlag, 1977.

[MN08] M. Mendel, A. Naor, Metric cotype, Ann. Math., 168 (2008), 247-298; arXiv:math/0506201.

[MS86] V.D. Milman, G. Schechtman, Asymptotic theory of finite dimensional normed spaces, Berlin, Springer-Verlag, 1986.

[Ost11a] M. I. Ostrovskii, On metric characterizations of some classes of Banach spaces, C. R. Acad. Bulgare Sci., 64 (2011), no. 6, 775-784; arXiv:1102.5082.

[Ost11b] M. I. Ostrovskii, Auerbach bases and minimal volume sufficient enlargements, Canadian Math. Bull., 54 (2011), 726-738; arXiv:1103.0997.

[Ost11c] M. I. Ostrovskii, Embeddability of locally finite metric spaces into Banach spaces is finitely determined, Proc. Amer. Math. Soc., posted on November 28, 2011, PII S 0002-9939(2011)11272-3 (to appear in print); arXiv:1103.0748.

[Ost11d] M. I. Ostrovskii, Different forms of metric characterizations of classes of Banach spaces, Houston J. Math., to appear; arXiv:1112.0801.

[OP75] R. I. Ovsepian, A. Pełczyński, On the existence of a fundamental total and bounded biorthogonal sequence in every separable Banach space, and related constructions of uniformly bounded orthonormal systems in $L^{2}$, Studia Math., 54 (1975), no. 2, 149-159.

[Pel76] A. Pełczyński, All separable Banach spaces admit for every $\varepsilon>0$ fundamental total and bounded by $1+\varepsilon$ biorthogonal sequences, Studia Math., 55 (1976), no. 3, 295-304.

[Pis86] G. Pisier, Probabilistic methods in the geometry of Banach spaces, in: Probability and analysis (Varenna, 1985), 167-241, Lecture Notes in Math., 1206, Springer, Berlin, 1986. 
[Pli76] A. Plichko, Existence of a complete $\varepsilon$-orthonormal system in a separable normed space (Russian), Dokl. Akad. Nauk Ukrain. SSR, Ser. A, 1976, no. 1, 22-23.

[Pli95] A. Plichko, On the volume method in the study of Auerbach bases of finite-dimensional normed spaces, Colloq. Math., 69 (1995), 267-270.

[Rib76] M. Ribe, On uniformly homeomorphic normed spaces, Ark. Mat., 14 (1976), no. 2, $237-244$.

[WS11] D.P. Williamson, D. B. Shmoys, The Design of Approximation Algorithms, Cambridge University Press, 2011.

[Yu06] G. Yu, Higher index theory of elliptic operators and geometry of groups, in: International Congress of Mathematicians, Vol. II, 1623-1639, Eur. Math. Soc., Zürich, 2006.

Department of Mathematics and Computer Science

ST. JOHN's UNIVERSITY

8000 Utopia Parkway, QueEns, NY 11439, USA

e-mail: ostrovsm@stjohns.edu 